\title{
Neovascular glaucoma: a retrospective review from a tertiary center in China
}

\author{
Na Liao, Chaohong Li, Huilv Jiang, Aiwu Fang, Shengjie Zhou and Qinmei Wang ${ }^{*}$
}

\begin{abstract}
Background: The purpose of this study is to report the prevalence, etiology, treatment and outcomes of neovascular glaucoma (NVG) in a tertiary care ophthalmic center in China.

Methods: Medical records of patients diagnosed as NVG at the Wenzhou Medical University between 2003 and 2014 were reviewed. Success was defined as IOP between 6 and $21 \mathrm{mmHg}$ without topical or systemic glaucoma medications with retention of presenting visual acuity (VA).

Results: NVG was diagnosed in 483 of 8306 (5.8 \%) of all glaucoma patients. Etiology is reported for all 310 eyes of 284 patients managed in the department. Interventions depended on insurance as well as personal finances; outcomes are reported for the 149 eyes of 138 patients with complete data that met follow up requirements. Diabetic retinopathy (DR,39.7 \%) was the major cause of NVG. Kaplan Meier survival analysis showed a success rate of $84.8 \%$ at 1 year, $47.5 \%$ at 3 years and $21.9 \%$ at 5 years. Major interventions included glaucoma drainage device (GDD) in 103 eyes and trans-scleral cyclophotocoagulation (TSCPC) in 22 eyes. Complications were more common in the GDD group.

Conclusions: NVG comprised $5.8 \%$ of glaucoma patients seen in a tertiary Chinese hospital. DR was identified as the commonest cause and probably reflects the increasing prevalence of diabetes in China. Surgical interventions were partly determined by insurance status and personal finances. GDD was the commonest surgical intervention used and also had the most complications.
\end{abstract}

Keywords: Neovascular glaucoma, Etiology, Glaucoma drainage device

\section{Background}

Neovascular glaucoma (NVG) is a secondary, refractory condition that accounts for $0.7-5.1 \%$ of glaucoma in an Asian population $[1,2]$. The condition is secondary to obstruction of the trabecular meshwork by neo-vascular membrane that develops in response to retinal ischemia $[3,4]$. Current surgical options for intraocular pressure (IOP) control in NVG comprise augmented trabeculectomy, glaucoma drainage devices (GDD) and cyclophotocoagulation (CPC) while prognostic factors include young age, previous vitrectomy and postsurgery complications [4-6]. Recent prospective data on the management of refractory glaucoma from China reported GDD as safe and effective for some of the conditions but with a relatively poor outcome in

\footnotetext{
* Correspondence: wqm3@mail.eye.ac.cn

School of Optometry and Ophthalmology and Eye Hospital, Wenzhou Medical University, Wenzhou, Zhejiang, China
}

NVG [7]. Late presentation and loss of follow up are additional challenges for the management of NVG in China [8].

There is paucity of data from China relating the causes and management of NVG. The objective of this study is to report the causes, management and outcomes of NVG in a tertiary facility in China.

\section{Methods}

The study was approved by the ethics committee of the Wenzhou medical university. As this was a retrospective study with de-identified data informed consent was not required. The records of all patients diagnosed as NVG between June 2003 and March 2014 at the eye hospital of Wenzhou Medical University, Wenzhou, China were reviewed. The diagnosis of NVG was based on an IOP > $21 \mathrm{mmHg}$ on applanation tonometry associated with neovascularization of the iris and/or angle of the anterior 
chamber detected by slit lamp bio-microscopy and gonioscopy [9]. All patients with NVG treated in our hospital were investigated to determine the cause. Other data extracted from the records included age, gender, affected eye, visual acuity(VA), lens status, IOP, number of glaucoma medications used at presentation and at last visit, types of intervention, history of previous intraocular surgery, use of anti-VEGF agents and post operative complications. Etiology of NVG was reported for all patients managed in the hospital. Patients with a follow up of less than 6 months were excluded, as were those without IOP and VA data at the final visit.

All patients received treatment for the underlying cause as well as medical treatment for control of IOP. Surgery was undertaken if further IOP lowering was deemed necessary either for retention of vision or for comfort. The overall management however depended not only on surgeon preference but also on insurance cover as well as capacity to pay. While $95 \%$ of our patients had medical insurance, reimbursement is not uniform and payment for prosthesis like GDD is usual.

Surgery if indicated was undertaken by one of 5 surgeons. For patients with useful vision, most surgeons preferred GDD as the first surgical option. Trabeculectomy was offered for such patients if economic factors precluded the use of a GDD, while trans-scleral cyclophotocoagulation (TSCPC) was generally offered those without useful vision. Endoscopic cyclophotocoagulation (Endo-CPC) was introduced in our hospital in 2006 and was combined with cataract surgery/vitrectomy or used in isolation for refractory NVG in some pseduophakic eyes. Anti-vascular endothelial growth factor (antiVEGF) became available in the clinic from 2008 and was used on a case-by-case basis determined primarily by insurance cover and affordability.

All GDD surgery was performed using Ahmed implants (New World Medical, Inc., Rancho Cucamonga, CA, USA). The surgical technique used for GDD and trabeculectomy were similar to that described in the literature [10, 11]. All trabeculectomies were performed with adjunctive mitomycin $C$, but the decision to use mitomycin with GDD was made by the individual surgeon. TSCPC was performed using the G probe with the diode laser (Iridex Corporation, Mountain View, CA, USA); Endo-CPC; was undertaken with the Endo-OPTIKS machine (Endo-OPTIKS, Little Silver, NJ, USA). The technique of TSCPC and Endo-CPC used was similar to that described [12, 13]. For TSCPC, three to four quadrants were treated with the $\mathrm{G}$ probe with about 20 spots avoiding the 3 and 9 o'clock positions with parameters adjusted to avoid a 'pop'. For Endo-CPC about 200 degrees of ciliary processes were coagulated.

Success was defined as an IOP between 6 and $21 \mathrm{mmHg}$ without topical or systemic glaucoma medications with retention of presenting VA; this visual criteria was applied to patients with a VA of light perception or better [5]. Snellen's VA was converted to the logarithm of the minimal angle of resolution (LogMAR) for analysis. An improvement or decrease in visual acuity was defined as a change of two or more lines on the LogMAR scale. LogMAR values for low vision were defined as follows: counting fingers $(\mathrm{CF})=2.3$; hand motions $(\mathrm{HM})=2.6$; light perception $(\mathrm{LP})=2.9$; and no light perception $(\mathrm{NLP})=4$ [14]. For those with low vision, a difference of one low vision category post-surgery was considered as change. Patients with incomplete data (lack of IOP and VA) and follow up less than 6 months were excluded from the analysis of outcomes.

\section{Statistical analysis}

All statistical analyses were performed using SPSS software version 15.0 (SPSS, Inc., Chicago, IL) and $p<0.05$ was considered significant. Normality of variables was ascertained using Kolmogorov-Smirnov tests. Continuous normally distributed data were presented as mean \pm standard deviation; proportions (\%) were used to describe categorical variables while non-normal distribution variables were presented as median (interquartile range). The paired student $t$-test and Wilcoxon signed-rank test was performed to compare the IOP and mean number of anti-glaucoma medications at presentation and at last visit respectively. Kaplan Meir survival analysis was used to report success rates. Cox's proportional hazards regression model [odds ratio (OR) with $95 \%$ confidence interval $(\mathrm{CI})]$ was used to report the association of potential prognostic factors: age, binocular involvement, lens status, previous intraocular surgery (vitrectomy or cataract surgery), systemic diseases, use anti-VEGF for underlying cause, history of PRP and influence of postoperative complications.

\section{Results}

NVG was diagnosed in a total of 483 (5.8 \%) of the 8306 glaucoma patients seen during the study period. 199 patients elected to seek care elsewhere while 284 were investigated and managed at the Wenzhou medical university eye department. Etiology of NVG was established and reported for these 284 patients. After excluding 14 patients lacking IOP or VA data at their last visit and 132 patients with less than 6 months follow up, 149 eyes of 138 patients ( 85 males and 53 females) were available for reporting interventions and outcomes.

Patient demographics are shown in Table 1. All patients were Han-Chinese. The mean age was $64.2 \pm$ 14.0 years (range, 10-94 years) and the median followup 18.5 months (range, 6.07-103.8 months). There were 105 phakic (70.5 \%), 11 aphakic $(7.4 \%)$ and 33 pseudophakic $(22.1 \%)$ eyes. The causes of NVG are listed in 
Table 1 Demographic Data

\begin{tabular}{|c|c|}
\hline \multicolumn{2}{|c|}{ Clinical characteristics of eyes with neovascular glaucoma $(N=149)$} \\
\hline Age in years (range) & $64.2 \pm 14.0(10-94)$ \\
\hline \multicolumn{2}{|l|}{ Eyes (\%) } \\
\hline Right & $76(51.0 \%)$ \\
\hline Left & $73(49.0 \%)$ \\
\hline \multicolumn{2}{|l|}{ Gender (\%) } \\
\hline Male & $85(61.6 \%)$ \\
\hline Female & $53(38.4 \%)$ \\
\hline \multicolumn{2}{|l|}{ Lens (\%) } \\
\hline Phakic & $105(70.5 \%)$ \\
\hline Aphakic & $11(7.4 \%)$ \\
\hline Pseudophakic & $33(22.1 \%)$ \\
\hline \multicolumn{2}{|l|}{ Presenting Vision } \\
\hline Improvement VA & $21(22.6 \%)$ \\
\hline Unchanged $^{a}$ & $34(36.6 \%)$ \\
\hline Decrease VA & $38(40.9 \%)$ \\
\hline Decrease to NLP & $16(17.2 \%)$ \\
\hline Previous $V A=1$ & 2 \\
\hline Previous VA $=\mathrm{CF}$ & 1 \\
\hline Previous $V A=H M$ & 6 \\
\hline Previous VA $=L P$ & 7 \\
\hline IOP at presentation ${ }^{\mathrm{b}}$ & $43.5 \pm 10.9$ \\
\hline IOP at final follow-up ${ }^{b}$ & $19.0 \pm 11.4$ \\
\hline \multicolumn{2}{|l|}{ Glaucoma medications } \\
\hline Median at presentation (IQR) & $2(3)$ \\
\hline Median at final follow up (IQR) & $0(1.5)$ \\
\hline Median interval of follow up in months (IQR) & $18.5(17.6)$ \\
\hline History of intraocular surgery & $54(36.2 \%)$ \\
\hline Complications & $38(25.5 \%)$ \\
\hline Enucleation (\%) & $5(3.4 \%)$ \\
\hline Hypotony (\%) & $8(5.4 \%)$ \\
\hline
\end{tabular}

$I O P$ intraocular pressure, $V A$ visual acuity, IQR interquartile range

${ }^{a} 56$ eyes with NLP at presentation were excluded

${ }^{b_{5}}$ enucleation and 2 phthisis bulbi excluded

Table 2. Diabetic retinopathy (123/310 eyes, $39.7 \%)$ was the commonest followed by CRVO (66/310 eyes, $21.3 \%$ ). The primary cause could not be determined in 59 eyes (19.0\%). 26 patients had bilateral NVG; 15 of these patients presented with bilateral NVG while 11 were diagnosed in the fellow eye after 1 to 16 months. 20/26 (76.2 \%) cases with bilateral NVG were caused by DR, two were due to bilateral CRVO, one had bilateral uveitis, one was ascribed to bilateral BRVO, one was caused by the ocular ischemia syndrome while the cause in one bilateral case remained unknown.

In eyes with presenting VA of LP or better (93 eyes of 86 patients), VA was retained in 34 eyes (36.6\%),
Table 2 Etiological Factors Associated with NVG

\begin{tabular}{lc}
\hline Causes of neovascular glaucoma & Eyes (\%) \\
\hline Retinal ischemic disease & $123(39.7)$ \\
DR & $66(21.3)$ \\
CRVO & $5(1.6)$ \\
BRVO & $3(1.0)$ \\
CRAO & $17(5.5)$ \\
RD & $9(2.9)$ \\
Others & a \\
Ocular ischemia syndrome & $7(2.3)$ \\
Uveitis & $6(1.9)$ \\
Trauma & $13(4.2)$ \\
Surgical causes & $3(1.0)$ \\
Radiation & $1(0.3)$ \\
Unknown & $59(19.0)$ \\
\hline $\begin{array}{l}\text { DR diabetic retinopathy, CRVO central retinal vein occlusion, CRAO central retinal } \\
\text { artery occlusion, BRVO branch retinal vein occlusion, RD retinal detachment }\end{array}$ \\
Including Coat's exudative retinopathy (4), Ischemic optic neuropathy (2), \\
Hypertensive retinopathy (1) and Persistent Hyperplastic Primary Vitreous (1) \\
and Eales' disease (1)
\end{tabular}

decreased in 38 eyes (40.9\%) and improved in 21 (22.6\%) eyes. Deterioration of vision to NLP occurred in 16/93 (17.2 \%) eyes. Two of these 16 eyes had useful VA (LogMAR $=1)$ prior to surgery. One case was caused by severe DR while the other was caused by BRVO. Both were managed with a GDD; one case developed retinal detachment.

Of the 149 eyes, two developed phthisis bulbi and 5 required enucleation. Excluding these 7 eyes, IOP decreased from a mean $( \pm$ SD) of $43.5 \pm 10.9$ to $19.0 \pm 11.4 \mathrm{mmHg}$ $(p=0.000)$. The number of glaucoma medications was reduced from 2 (interquartile range $=3$ ) to 0 (interquartile range $=1.5)$ after interventions $(p=0.000)$

Kaplan-Meier survival analysis is shown in Fig. 1. Success was achieved in $84.8 \%$ at 1 year, $47.5 \%$ at 3 years and $21.9 \%$ at 5 years. Six eyes that required combinations of GDD, TSCPC or trabeculectomy were excluded from this analysis (3 eyes that underwent TSCPC followed by GDD or Trabeculectomy and 3 that required TSCPC following GDD). Of the remaining 143 eyes, 103 eyes underwent GDD, 22 eyes were treated with TSCPC, 7 eyes had trabeculectomy, 3 eyes underwent Endo-CPC and 8 eyes underwent combined surgery. Table 3 details the treatment modalities and their outcomes. Age, lens status, the use of anti-VEGF, postoperative complications, PRP, prior-intraocular surgery did not have a statistically significant influence on outcome (Table 4).

Postoperative complications are summarized in Table 5. Shallow anterior chamber was the commonest complication $(12 / 143,8.4 \%)$ followed by hypotony $(8 / 143$, $5.6 \%)$. Tube-related complications included occlusion/ 


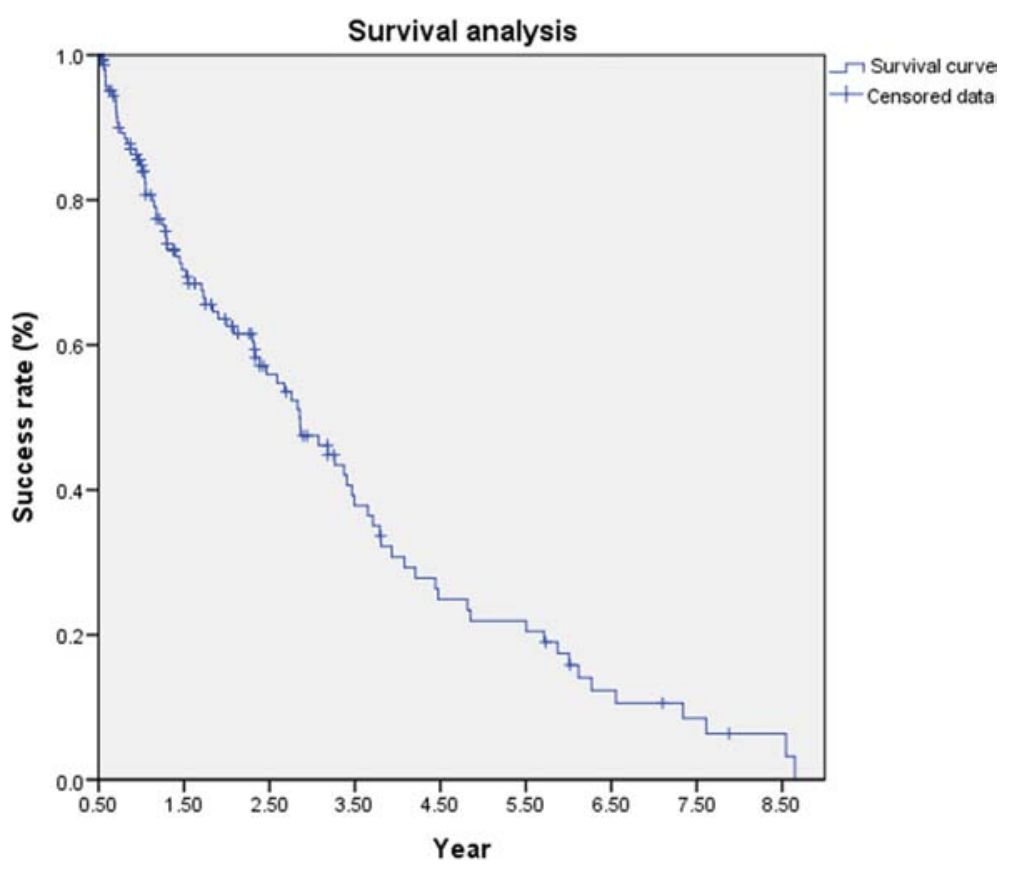

Fig. 1 Kaplan-Meier survival curve of treatment outcomes in all 138 NVG patients (149 eyes). Censored data relates to the ones who had not observed failure at that time (including missing data)

displacement of the tube and occurred in 2 eyes. Most complications occurred in the GDD group.

\section{Discussion}

A survey by the Beijing union medical college hospital and WHO in 1996 reported that the prevalence of secondary glaucoma in the population over the age of 50 years was $0.12 \%$ and accounted for $5.9 \%$ of all glaucoma [15]. NVG per se was not reported, but as it is a secondary glaucoma, we can infer that the proportion of NVG in that survey was lower than $5.9 \%$. NVG was diagnosed in $5.8 \%$ of 8306 glaucoma patients seen in our tertiary hospital. This is similar to the $6.7 \%$ of 1232
Chinese subjects diagnosed as glaucoma in a population based survey in Singapore [16]. Although it cannot really be compared to population-based data, Our data provides the prevalence of NVG in a tertiary eye hospital in China.

DR (39.7 \%) followed by CRVO (21.3 \%) was the major cause of NVG in this series. While the etiology could only be determined for the 284 patients who elected to continue their care in our center, the pattern is consistent with reports from other countries [17-19]. A recent hospital based report from China provides conflicting results: CRVO (39.2 \%) was reported as the commonest cause in 120 NVG eyes [20]. Diabetes, a major cause of NVG has

Table 3 Treatment and results $(N=143 \text { eyes })^{a}$

\begin{tabular}{lllll}
\hline Treatments[N(\%)] & No. cases & Complete success rate & Decreased VA & The percentage of IOP reduction \\
\hline TB & $7(4.9 \%)$ & $4(57.1 \%)$ & $2(28.6 \%)$ & $70.0 \%$ \\
GDD & $103(72.0 \%)$ & $46(32.2 \%)$ & $35(34.0 \%)$ & $54.8 \%^{\mathrm{b}}$ \\
TSCPC & $22(15.4 \%)$ & $7(31.8 \%)$ & $4(18.2 \%)$ & $66.8 \%^{\mathrm{c}}$ \\
Endo-CPC & $3(2.1 \%)$ & 0 & $3(100 \%)$ & $17.2 \%^{\mathrm{d}}$ \\
Combined surgery & $8(5.6 \%)$ & $4(50.0 \%)$ & $1(12.5 \%)$ & $61.1 \%$ \\
Vitrectomy \& GDD & 3 & 1 & 0 & $48.2 \%$ \\
Cataract \& GDD & 3 & 3 & 0 & $73.6 \%$ \\
Cataract \& Endo-CPC & 2 & 0 & 1 & $60.5 \%$
\end{tabular}

TB trabeculectomy, GDD glaucoma drainage devices, TSCPC trans sclera cyclophotocoagulation, Endo-CPC endoscopic cyclophotocoagulation ${ }^{a} 6$ eyes treated with multiple operations excluded

${ }^{\mathrm{b}} \mathrm{GDD}$ group excludes 1 enucleated eye

'TSCPC group excludes 3 enucleated eyes and 1 with phthisis bulbi

${ }^{d}$ Endo-CPC group excludes 1 eye with phthisis 
Table 4 Prognostic factors

\begin{tabular}{llcl}
\hline Parameters & $P$ & Odds ratio & 95 \% confidence interval \\
\hline Age & 0.85 & 1.07 & $0.53-2.16$ \\
Binocular & 0.34 & 1.36 & $0.72-2.56$ \\
Lens status & 0.99 & 1.00 & $0.72-1.39$ \\
Prior surgery & 0.78 & 1.10 & $0.59-2.02$ \\
Hypertension & 0.34 & 1.28 & $0.77-2.11$ \\
Diabetes & 0.18 & 0.69 & $0.40-1.19$ \\
Anti-VEGF & 0.32 & 1.79 & $0.58-5.56$ \\
Complications & 0.30 & 0.78 & $0.48-1.25$ \\
PRP & 0.43 & 1.22 & $0.74-2.01$ \\
\hline
\end{tabular}

increased in China over the years and has a current reported prevalence of $23 \%$, affecting an estimated 92.4 million adults in the mainland [21]. Our data seems to suggest a change in etiology of NVG that is likely linked to the increasing prevalence of diabetes in China. Lack of data and the retrospective nature of the study contributed to our inability to identify the cause in $19 \%$ of cases.

VA results were sobering. Acuity was poor to start with and could only be preserved or improved in $59 \%$ of cases while $41 \%$ worsened, with 16 eyes deteriorating to no light perception. Improvement in vision occurred in $21 / 93$ eyes (90 \% due to resolution of corneal edema, hyphema and/or vitreous hemorrhage). GDD was the commonest surgical intervention used and also had the most complications. Interventions in our hospital depend on surgeon preference and experience as well as cover by medical insurance. GDD (103/143, $70.2 \%)$ was the first option for controlling IOP in NVG patients with potential for vision. TSCPC was the second choice (22/ $143,15.4 \%)$ and tended to be used in those with a poorer prognosis.

Kaplan-Meier survival analysis showed a 84.8 probability of success at 1 year, $62.6 \%$ at 2 years and $21.9 \%$ at 5 years.

Table 5 Postoperative complications ( $N=143$ eyes) ${ }^{a}$

\begin{tabular}{|c|c|c|}
\hline Complications & Number (\%) & $\begin{array}{l}\text { Group with highest } \\
\text { incidence }\end{array}$ \\
\hline Hyphema & $4(2.8)$ & GDD (4) \\
\hline Shallow anterior chamber & $12(8.4)$ & GDD (10) \\
\hline Choroidal detachment & $2(1.4)$ & GDD (2) \\
\hline Ciliary body detachment & $4(2.8)$ & GDD (4) \\
\hline Tube related complications ${ }^{b}$ & $2(1.9)$ & - \\
\hline Bleb-related complications ${ }^{c}$ & $3(2.6)$ & GDD (3) \\
\hline Hypotony & $8(5.6)$ & GDD (4) \\
\hline Endophthalmitis & 0 & - \\
\hline
\end{tabular}

GDD glaucoma drainage devices

${ }^{a} 6$ eyes treated with multiple operations were excluded

${ }^{b} 103$ eyes treated with GDD were analyzed

' 22 eyes treated with TSCPC, 3 with Endo-CPC and 2 with cataract \& Endo-CPC were excluded
Nakatake, etc. found that success rate of primary trabeculectomy (with or without anti-VEGF) prior to surgery was $70.9 \%$ after 1 year, decreasing to $60.8 \%$ in 2 years [6]. A recent report from Japan showed that the success rate was higher ( $83 \%$ at 3 years) if trabeculectomy was preceded by a preoperative intravitreal injection of Bevacizumab [22]. A publication from Korea reported a success rate of $79 \%$ at 1 year decreasing to less than $60 \%$ at 2 years [23]. As our numbers were small we could not report the outcomes for each intervention separately and our results cannot be easily compared to previous reports. However our findings do reflect the reality of management, outcome and poor follow up even in a tertiary care center China. The high success rate at 1 year could be due to inclusion criteria, exclusion of patients with follow up less than 6 months as well as loss to follow up.

Cox proportional hazards model showed that factors like systemic disease, lens status, complications, antiVEGF agents, PRP and prior-intraocular surgery did not influence the treatment outcome of NVG, but our numbers are small. While, anti-VEGF agents may be beneficial for the management of NVG reducing the need for glaucoma surgery and decreasing complications in eyes with neovascular glaucoma, the addition of intravitreal bevacizumab did not seem to increase the success rate in previous studies [24, 25]. All cases that required PRP underwent the procedure, but its influence on incidence of NVG is not clear $[26,27]$. While PRP eliminates the stimulus for neovascularization it does not affect existing peripheral anterior synechiae (PAS) [28].

The complications encountered following intervention in this study were those usually reported after such interventions. Shallow anterior chamber was the most frequent early complication (8.5\%), while hypotony was the most frequent late complication (5.6\%). The extreme low complication rate could not compare with previous reports from Asian directly [6, 29]. It is very likely that the discrepancy between complication rates in our study and others are related to the poor follow up rates in the current study (i.e. patients with mild complications mostly like lost follow-up) and some data unavailable in this retrospective study. Given the limitations of sample size we too found that larger IOP reductions could be achieved with tube surgery compared to TSCPC but the postoperative complications were higher and included some sight threatening ones [30]. Although we did not encounter any endophthalmitis, phthisis and severe pain requiring enucleation occurred in 5 patients, three of those in the TSCPC group.

The limitations of the study include the retrospective design, exclusion of a large number of cases due to lack of follow up and lack of data as well as the lack of standardized management. The lack of a preferred practice 
or standardized regime also meant that the numbers for some interventions were too small to conduct any analysis. The fact that the management is affected by insurance status and personal finances made it difficult to create a protocol or standardize procedures, but this as well as poor follow up is the reality in China. This case series does however demonstrate the changing etiology of NVG in China, has the longest follow-up of NVG in an Asian population and reflects the reality of management in China.

\section{Conclusion}

Diabetic retinopathy was the commonest detected cause of NVG encountered in a tertiary hospital in China. Cases present late, follow up is poor and management is partly determined by surgeon preference, insurance cover and personal finances. The increasing prevalence of diabetes in China will likely lead to a higher incidence of NVG and other related complications.

\section{Competing interests}

The authors declare that they have no competing interests.

\section{Authors' contributions}

NL was responsible for, collection of data, analysis and interpretation of results and wrote the first draft of the manuscript. $\mathrm{CHL}$ participated in its design and helped to draft the manuscript. HLJ was involved in data collection. AWF and SJZ helped perform the analysis; QMW conceived the study. All authors read and approved the final manuscript.

\section{Acknowledgements}

This work was supported in part by the National Science and Technology Ministry (2012BAI08B04); National Natural Science Foundation of China (81300804); the Scientific Research Fund of Wenzhou Medical University (QTJ11022).

\section{Received: 16 May 2015 Accepted: 13 January 2016}

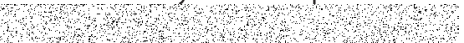

\section{References}

1. Wong TY, Chong EW, Wong WL, Rosman M, Aung T, Loo JL, et al. Prevalence and causes of low vision and blindness in an urban malay population: the Singapore Malay Eye Study. Arch Ophthalmol. 2008;126(8):1091-9.

2. Narayanaswamy A, Baskaran M, Zheng Y, Lavanya R, Wu R, Wong WL, et al. The prevalence and types of glaucoma in an urban Indian population: the Singapore Indian Eye Study. Invest Ophthalmol Vis Sci. 2013;54(7):4621-7.

3. Brown GC, Magargal LE, Schachat A, Shah H. Neovascular glaucoma. Etiologic considerations. Ophthalmology. 1984;91(4):315-20.

4. Sivak-Callcott JA, O'Day DM, Gass JD, Tsai JC. Evidence-based recommendations for the diagnosis and treatment of neovascular glaucoma. Ophthalmology. 2001;108(10):1767-76. quiz1777, 1800.

5. Takihara $Y$, Inatani M, Fukushima M, Iwao K, Iwao M, Tanihara H. Trabeculectomy with mitomycin C for neovascular glaucoma: prognostic factors for surgical failure. Am J Ophthalmol. 2009;147(5):912-8. 918 e911.

6. Nakatake S, Yoshida S, Nakao S, Arita R, Yasuda M, Kita T, et al. Hyphema is a risk factor for failure of trabeculectomy in neovascular glaucoma: a retrospective analysis. BMC Ophthalmol. 2014;14:55.

7. Li Z, Zhou M, Wang W, Huang W, Chen S, Li X, et al. A prospective comparative study on neovascular glaucoma and non-neovascular refractory glaucoma following Ahmed glaucoma valve implantation Chin Med J (Engl). 2014;127(8):1417-22.

8. Zhang X, Zhou M. Neovascular glaucoma: challenges we have to face. Chin Med J (Engl). 2014;127(8):1407-9.
9. Netland PA. The Ahmed glaucoma valve in neovascular glaucoma (An AOS Thesis). Trans Am Ophthalmol Soc. 2009;107:325-42.

10. Oguri A, Takahashi E, Tomita G, Yamamoto T, Jikihara S, Kitazawa Y. Transscleral cyclophotocoagulation with the diode laser for neovascular glaucoma. Ophthalmic Surg Lasers. 1998;29(9):722-7.

11. Takada S, Hoshino $Y$, Ito H, Masugi $Y$, Terauchi T, Endo $K$, et al. Extensive bowel necrosis related to bevacizumab in metastatic rectal cancer patient: a case report and review of literature. Jpn J Clin Oncol. 2015;45(3):286-90.

12. Uram M. Endoscopic cyclophotocoagulation in glaucoma management Curr Opin Ophthalmol. 1995;6(2):19-29.

13. Zhang SH, Dong FT, Mao J, Bian AL. Factors related to prognosis of refractory glaucoma with diode laser transscleral cyclophotocoagulation treatment. Chin Med Sci J. 2011;26(3):137-40.

14. Sidoti PA, Dunphy TR, Baerveldt G, LaBree L, Minckler DS, Lee PP, et al. Experience with the Baerveldt glaucoma implant in treating neovascular glaucoma. Ophthalmology. 1995;102(7):1107-18.

15. Zhao J, Sui R, Jia L, Ellwein LB. Prevalence of glaucoma and normal intraocular pressure among adults aged 50 years or above in Shunyi county of Beijing. Zhonghua Yan Ke Za Zhi. 2002;38(6):335-9.

16. Foster PJ, Oen FT, Machin D, Ng TP, Devereux JG, Johnson GJ, et al. The prevalence of glaucoma in Chinese residents of Singapore: a cross-sectional population survey of the Tanjong Pagar district. Arch Ophthalmol. 2000;118(8):1105-11.

17. Al-Shamsi HN, Dueker DK, Nowilaty SR, Al-Shahwan SA. Neovascular glaucoma at king khaled eye specialist hospital - etiologic considerations. Middle East Afr J Ophthalmol. 2009:16(1):15-9.

18. Shen CC, Salim S, Du H, Netland PA. Trabeculectomy versus Ahmed Glaucoma Valve implantation in neovascular glaucoma. Clin Ophthalmol. 2011;5:281-6.

19. Woodcock MG, Richards JC, Murray AD. The last 11 years of Molteno implantation at the University of Cape Town. Refining our indications and surgical technique. Eye (Lond). 2008;22(1):18-25.

20. Liu L. Etiology analysis of neovascular glaucoma in 120 cases. Int J Ophthalmol. 2011;11(3):521-3.

21. Yang SH, Dou KF, Song WJ. Prevalence of diabetes among men and women in China. N Engl J Med. 2010;362(25):2425-6. author reply 2426.

22. Kobayashi S, Inoue M, Yamane S, Sakamaki K, Arakawa A, Kadonosono K. Long-term Outcomes After Preoperative Intravitreal Injection of Bevacizumab Before Trabeculectomy for Neovascular Glaucoma. J Glaucoma. 2015 Jan 9. doi:10.1097/IJG.0000000000000211.

23. Lee HY, Park JS, Choy YJ, Lee HJ. Surgical outcomes of different Ahmed Glaucoma Valve implantation methods between scleral graft and scleral flap. Korean J Ophthalmol. 2011;25(5):317-22.

24. Wakabayashi T, Oshima Y, Sakaguchi H, Ikuno Y, Miki A, Gomi F, et al. Intravitreal bevacizumab to treat iris neovascularization and neovascular glaucoma secondary to ischemic retinal diseases in 41 consecutive cases. Ophthalmology. 2008;115(9):1571-80. 1580 e1571-1573.

25. Fong AW, Lee GA, O'Rourke $P$, Thomas R. Management of neovascular glaucoma with transscleral cyclophotocoagulation with diode laser alone versus combination transscleral cyclophotocoagulation with diode laser and intravitreal bevacizumab. Clin Experiment Ophthalmol. 2011;39(4):318-23.

26. Striga M, Ivanisevic M. Comparison between efficacy of full- and mild-scatter (panretinal) photocoagulation on the course of diabetic rubeosis iridis. Ophthalmologica. 1993;207(3):144-7.

27. Hayreh SS, Klugman MR, Podhajsky P, Servais GE, Perkins ES. Argon laser panretinal photocoagulation in ischemic central retinal vein occlusion. A 10-year prospective study. Graefes Arch Clin Exp Ophthalmol. 1990;228(4):281-96.

28. Ehlers JP, Spirn MJ, Lam A, Sivalingam A, Samuel MA, Tasman W. Combination intravitreal bevacizumab/panretinal photocoagulation versus panretinal photocoagulation alone in the treatment of neovascular glaucoma. Retina. 2008;28(5):696-702.

29. Yildirim N, Yalvac IS, Sahin A, Ozer A, Bozca T. A comparative study between diode laser cyclophotocoagulation and the Ahmed glaucoma valve implant in neovascular glaucoma: a long-term follow-up. J Glaucoma. 2009;18(3):192-6.

30. Bloom PA, Clement $\mathrm{Cl}$, King A, Noureddin B, Sharma K, Hitchings RA, et al. A comparison between tube surgery, ND:YAG laser and diode laser cyclophotocoagulation in the management of refractory glaucoma. Biomed Res Int. 2013;2013:371951. 